\title{
Development of A Thermodynamically-Consistent Alpha Function for the Patel-Teja-Valderrama Equation of State
}

\author{
Allan Paolo L. Almajose and Maria Lourdes P. Dalida
}

\begin{abstract}
A new, four-parameter alpha function with thermodynamically consistent parameter values is developed for predicting vapor pressures using the Patel-Teja-Valderrama equation of state. The form of the alpha function was derived by keeping in mind the thermodynamic consistency rules as provided by the limiting conditions in the determination of the generalized parameters in a generic cubic equation of state. Using MATLAB, codes executing a nonlinear program that would minimize errors between DIPPR-estimated vapor pressures between the triple point until the critical point from the alpha function's vapor pressure prediction has been developed. Thermodynamically consistent parameters were calculated by setting up nonlinear constraints for the derivatives, assuring a monotonically decreasing behavior for the function. The performance of the model was compared with five other models commonly used in industries and process simulation programs and is found to provide better accuracy in comparison when working with polar fluids. Further, its performance is found to be comparable to some models when estimating nonpolar and light fluids. The statistical analyses used to verify the performance of the model in comparison with the other models used in literature include the calculation of the r-squared, adjusted r-squared, predicted r-squared, absolute average deviation, root mean square errors, and by visual inspection. The study also included the determination of thermodynamically consistent parameter values for twenty different fluids commonly used in process simulations.
\end{abstract}

Index Terms-Alpha function, error minimization, energy parameter, double exponential, mathematical modelling, equation of state.

\section{INTRODUCTION}

Equations of state (EOS) are of great importance in the field of chemical engineering design, most especially in fluid phase equilibria. A large number of EOS have been proposed in literature, having either empirical, semi-empirical, or theoretical bases [1]. Thermodynamically, a cubic equation of state is an equation that represents a fluid's behavior over a wide pressure, volume, and temperature (PVT) range. Following the Van der Waals model, it assumes that the pressure of a fluid is exerted by the sum of the pressures exerted due to attraction and repulsion. Current works in improving the accuracy of cubic equations of state involve (1) modifying the repulsive term; and (2) modifying the attractive term [2]. The attraction parameter (typically

Manuscript received February 7, 2019; revised April 2, 2019. This work was supported in part by the Philippine Department of Science and Technology - Engineering Research and Development for Technology.

Allan Paolo L. Almajose is with the National Graduate School of Engineering, University of the Philippines-Diliman, Quezon City $1101 \mathrm{M}$. M., Philippines (e-mail: allan_paolo.almajose@upd.edu.ph).

Maria Lourdes P. Dalida is with the Department of Chemical Engineering, University of the Philippines-Diliman, Quezon City 1101 M. M., Philippines (e-mail: mpdalida@up.edu.ph). denoted as $\boldsymbol{a}$ ) is important as it defines the intermolecular forces in between the molecules of a chemical compound. In modifying the attractive term, either the general form by adding more EOS parameters in the attraction term or by modifying the attraction parameter itself; which contains the pure component energy parameter or generally known as the 'alpha function'. Modifying the alpha function is done to achieve predictions of higher accuracy. As with the strategy of Soave [3], modifying the alpha function of the attractive parameter greatly improved the vapor and liquid densities of the Redlich-Kwong EOS. Soave was able to achieve this by modifying the Redlich-Kwong energy parameter's alpha function. While a large amount of alpha functions has been proposed in literature, the equations however present a problem in thermodynamic consistency. Thermodynamic consistency in the development of an alpha function is important as functions with thermodynamic inconsistencies develop abnormal predictions when extrapolated at supercritical regions, or generate physically meaningless quantities when applied to mixture thermodynamics [4] Therefore, in order to provide greater accuracy in process simulations, an alpha function with thermodynamically consistent behavior must be derived.

\section{A. Significance}

Accurate vapor pressure prediction of pure components is an important prerequisite for both accurate calculations of thermodynamic parameters and vapor-liquid equilibria when using cubic EOS. As the vapor pressure of a pure component is directly related to the attractive term of an EOS, which is also directly related to the alpha function, a good alpha function that fits vapor pressures accurately is very important. Accurate thermodynamic calculations resulting from this study will be very useful in conducting process simulations and in equipment design especially in gas and fuel industries.

\section{METHODS}

\section{A. Vapor-Pressure Datasets}

The vapor pressure datasets were generated from the Design Institute for Physical Properties (DIPPR) equations for the vapor pressure. The DIPPR equations for the vapor pressure are highly reliable and is therefore used for this study [5]. In addition, the DIPPR equations provide accurate estimation of the vapor pressure from the triple point (as $\boldsymbol{T}_{\boldsymbol{m i n}}$ ) until the critical point (as $\boldsymbol{T}_{\boldsymbol{m a x}}$ ). The vapor pressure equation is defined in equation (1).

$$
\ln \left(P^{o}\right)=C_{1}+\frac{C_{2}}{T}+C_{3} \ln (T)+C_{4} T^{C_{5}}
$$




\section{B. Code Development, Thermodynamics, and Mathematics}

In this study, the Patel-Teja-Valderrama equation of state (PTVEOS) [2] is utilized in order to predict the vapor and liquid densities of the chemical compounds to be modeled. The PTVEOS is defined by equations (2) to (8).

$$
\begin{gathered}
P=\frac{R T}{V-b}-\frac{a}{V \cdot(V+b)+c \cdot(V-b)} \\
a=\Omega_{A} \frac{R^{2} T_{C}{ }^{2}}{P_{C}} \alpha(T) \\
b=\Omega_{B} \frac{R T_{C}}{P_{C}} \\
c=\Omega_{C} \frac{R T_{C}}{P_{C}} \\
\Omega_{A}=0.66121-0.76105 Z_{C} \\
\Omega_{B}=0.02207+0.20868 Z_{C} \\
\Omega_{C}=0.57765-1.87080 Z_{C}
\end{gathered}
$$

Parameters $\boldsymbol{a}, \boldsymbol{b}$, and $\boldsymbol{c}$ are substance-dependent constants and are functions of the critical temperature and pressure of the compound. The cubic equation is by recasting the cubic equation in terms of compressibility factor as shown in equations (9) to (12) to follow a standard cubic equation's formulation.

$$
\begin{gathered}
Z^{3}+(C-1) \cdot Z^{2}+\left(A-2 B C-B^{2}-(B+C)\right) \cdot Z \\
+\left(B^{2} C+B C-A B\right)=0
\end{gathered}
$$

where;

$$
\begin{gathered}
A=\frac{a P}{(R T)^{2}} \\
B=\frac{b P}{R T} \\
C=\frac{c P}{R T}
\end{gathered}
$$

The liquid and vapor fugacities are then calculated by integrating the equation using the pressure-explicit formulation of the fugacity, evaluated at both the vapor and liquid roots. The integrated fugacity equation of PTV EOS for pure components is defined by equations (13) to (14).

$$
\begin{array}{r}
\ln \left(\varphi^{P}\right)=Z^{P}-1-\ln |Z-B| \\
+\frac{a}{2 R T \Psi} \ln \mid \frac{Z^{P}+\left(\frac{b+c}{2}-\Psi\right) \frac{P}{R T}}{Z^{P}+\left(\frac{b+c}{2}+\Psi\right) \frac{P}{R T} \mid} \\
\Psi=\sqrt{b c+\left(\frac{b+c}{2}\right)^{2}}
\end{array}
$$

The superscript $\boldsymbol{P}$ corresponds to the phase of the compound, either liquid or vapor. The equilibrium relation is achieved by solving for the liquid and vapor fugacities.
In order to achieve the thermodynamic consistency of the proposed alpha function and the other functions included in this study for comparison, equispaced temperature points are used to evaluate the numerical first, second, and third derivatives calculated using five-point central finite difference formulations. The temperature points are selected in order to assure that the triple point and the critical point for the vast majority of compounds are covered. The central five-point formulation for the numerical first, second, and third derivative is defined in equations (15) to (17).

$$
\begin{gathered}
f_{2}^{\prime} \approx \frac{-f_{4}+8 f_{3}-8 f_{1}+f_{0}}{12 h}+O\left(h^{4}\right) \\
f_{2}^{\prime \prime} \approx \frac{-f_{4}+16 f_{3}-30 f_{2}+16 f_{1}-f_{0}}{12 h^{2}}+O\left(h^{4}\right) \\
f_{2}^{\prime \prime \prime} \approx \frac{f_{4}-2 f_{3}+2 f_{1}-f_{0}}{2 h^{3}}+O\left(h^{4}\right)
\end{gathered}
$$

The five-point stencil is used due to its inherent symmetric formulation compared to the four-point formula, which can also calculate the third derivative. The derivative in each temperature is translated into a vector, which represents the nonlinear constraint that will be used in the error minimization.

The objective function of the minimization algorithm is evaluated by calculating for the mean square error of the DIPPR vapor pressure prediction and the PTVEOS in conjunction with the alpha function used, given initial parameter values. The general nonlinear program for the study is shown in equation (18).

$$
\min _{\tau} P_{E R R}=\sum_{i}\left\lfloor\frac{\left(P_{i E O S}^{O}-P_{i}^{O}{ }_{D I P P R}\right)^{2}}{n}\right\rfloor
$$

In the nonlinear program, $\boldsymbol{n}$ is the number of assumed data points for modelling. It is important to remember that the vapor pressure as predicted by the equation of state is a function of the alpha function's parameters, represented by $\boldsymbol{\tau}$; a vector of alpha function parameters, as in equation (19), which is then subject to the nonlinear constraints as shown in equation (20).

$$
\begin{gathered}
P_{E O S}^{O}=f(\boldsymbol{\tau}) \\
\text { s.t. } \begin{cases}\frac{d(a)}{d T}<0 & 0^{+}<T<2000 \\
\frac{d^{2}(a)}{d T^{2}}>0 & 0^{+}<T<2000 \\
\frac{d^{3}(a)}{d T^{3}}<0 & 0^{+}<T<2000\end{cases}
\end{gathered}
$$

\section{Model Development and Statistical Verification}

The vapor pressure datasets were generated from the Design Institute for Physical Properties (DIPPR) equations for the vapor pressure. The DIPPR equations for the vapor pressure are highly reliable and is therefore used for this study [5]. In addition, the DIPPR equations provide accurate estimation of the vapor pressure from the triple point (as $\boldsymbol{T}_{\boldsymbol{m i n}}$ ) until the critical point (as $\boldsymbol{T}_{\boldsymbol{m a x}}$ ). The vapor pressure equation 
is defined as (1), whose constants can be found in the Perry's Chemical Engineers' Handbook.

According to thermodynamic rules in consistency of the energy parameter, it must be: Equal to unity when evaluated at the critical temperature;

1) Positive, and continuous for all positive temperature values;

2) Approaching zero at infinite temperature.

3) Negative, and continuous at the first derivative for all positive temperature values;

4) Positive, and continuous at the second derivative for all positive temperature values;

5) Negative at the third derivative for all positive temperature values.

As the alpha function must be alternatingly positive and negative for each derivative, the function should be a convex function; polynomial or exponential functions should work in modelling the energy parameter. However, the last condition states that the function must approach zero at infinite temperature. The final proposed model and the other models included in this study is shown in Table I.

TABLE I: MODELS INCLUDED IN THE STUDY

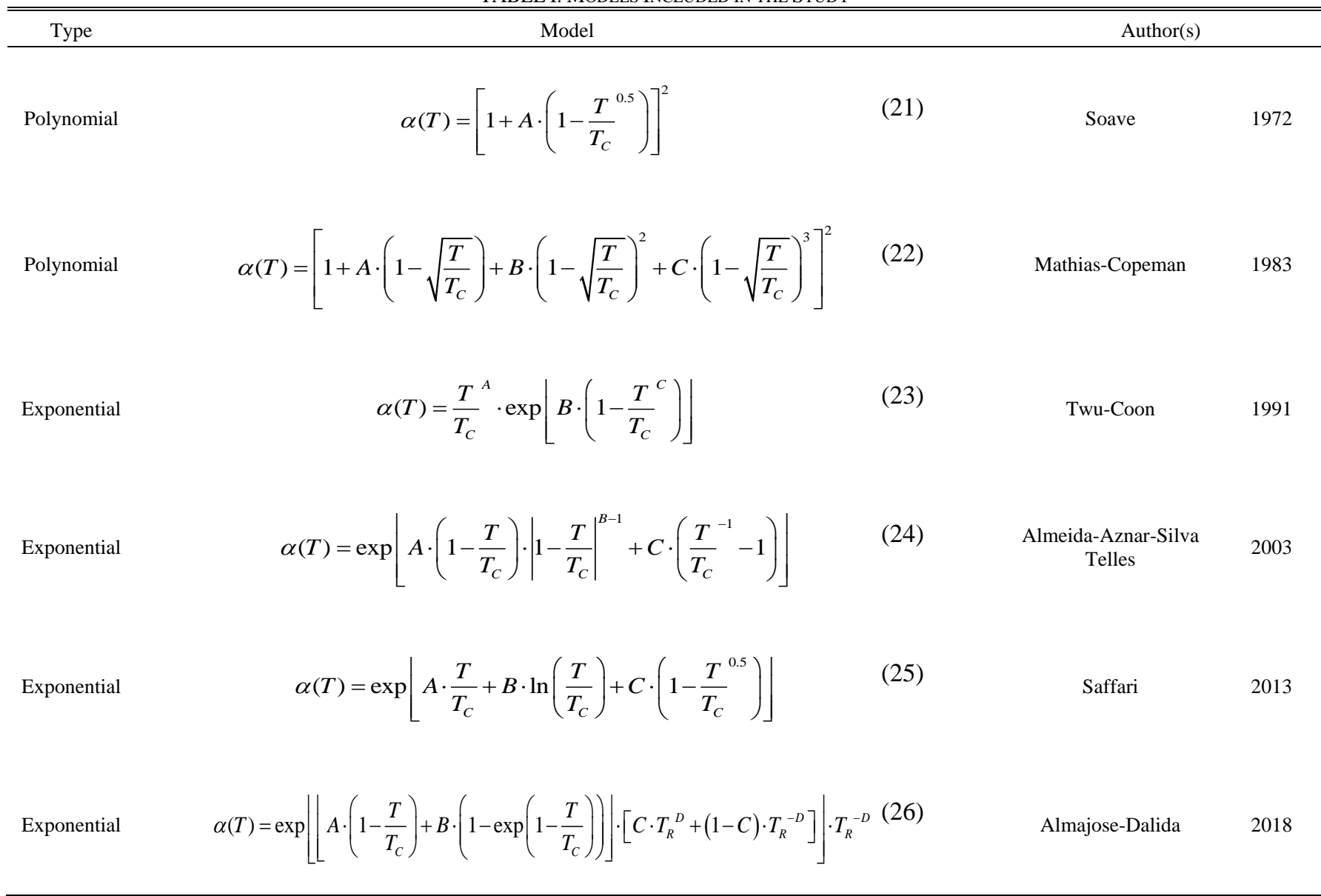

To determine the performance of the proposed model with respect to the other models available in literature in predicting the vapor pressure curve, statistical analyses used include the calculation of the R-squared, adjusted R-squared, predicted R-squared, and absolute average deviation. The formulation of the R-squared is shown as equation (27).

$$
R^{2}=1-\frac{\sum_{i}\left(P_{i \text { PTV }}^{O}-P_{i}^{O}{ }_{D I P P R}\right)^{2}}{\sum_{i}\left(P_{i \text { DIPPR }}^{O}-\bar{P}_{D I P P R}^{O}\right)^{2}}
$$

$\boldsymbol{P}_{\boldsymbol{i}}^{\boldsymbol{O}}{ }_{\boldsymbol{P} T \boldsymbol{V}}$ and $\boldsymbol{P}_{\boldsymbol{i}}^{\boldsymbol{O}}{ }_{\text {DIPPR }}$ represent the vapor pressure predictions of both PTVEOS and DIPPR correlations for each temperature point, and $\overline{\boldsymbol{P}}_{\boldsymbol{i}}^{\boldsymbol{O}}{ }_{\boldsymbol{D I P P R}}$ is the mean value of all DIPPR vapor pressure correlations for each temperature point.

The adjusted $R$-squared $\left(R^{2}\right.$-adj) calculation follows the formulation of SPSS as demonstrated by equation (28);

$$
R_{A D J}^{2}=1-\frac{\left(1-R^{2}\right)(i-1)}{i-n-1}
$$

Variable $\boldsymbol{i}$ corresponds to the number of vapor pressure temperature points; $\boldsymbol{n}$ is the number of variables in the regression equation, and $\boldsymbol{R}^{2}$ is the correlation coefficient as calculated previously by R-squared.

Calculation of the predicted $\mathrm{R}$-squared $\left(\mathrm{R}^{2}\right.$-pred) requires the generation of new vapor pressure points from both the DIPPR equations and the fitted equations, and R-squared is calculated as in equation (27).

The formulation of the absolute average deviation (AAD) is as follows:

$$
A A D=\frac{\sum_{i}\left|P_{i}^{O}{ }_{P T V}-P_{i}^{O}{ }_{D I P P R}\right|}{i}
$$


here values for $\boldsymbol{P}_{\boldsymbol{i}}^{\boldsymbol{O}} \boldsymbol{P T V}_{\boldsymbol{V}}$ and $\boldsymbol{P}_{\boldsymbol{i}}^{\boldsymbol{O}}{ }_{\boldsymbol{D I P P R}}$ are identical with the R-squared formulation.

\section{RESULTS}

Results show that the proposed model is thermodynamically consistent and is effective in predicting vapor pressure. It is also of notice that the Twu-Coon model and the Saffari model performed well in predicting vapor pressures. While the Soave, Almeida-Aznar-Silva Telles and the Mathias-Copeman models predict vapor pressures with varying accuracy, it is important to note that they are not thermodynamically consistent. This is due to the fact that the Soave and Mathias-Copeman equations follow a polynomial model, which does not terminate to zero at infinite temperatures. The Almeida-Aznar-Silva Telles is discontinuous at the critical temperature; in order to predict the vapor pressures correctly, a numerical limit is applied by adding $10^{-15}$ to the value of the temperature.

Further, as the value of the alpha function must be equal to 1 at the critical temperature, the Saffari function may only be thermodynamically consistent if the first parameter is equal to zero. If the first parameter takes a nonzero value, the entire exponential term not be equal to zero; in effect, the alpha function will not be strictly equal to 1 . Therefore, in order for the Saffari function to be thermodynamically consistent, the first parameter must be equal to zero.

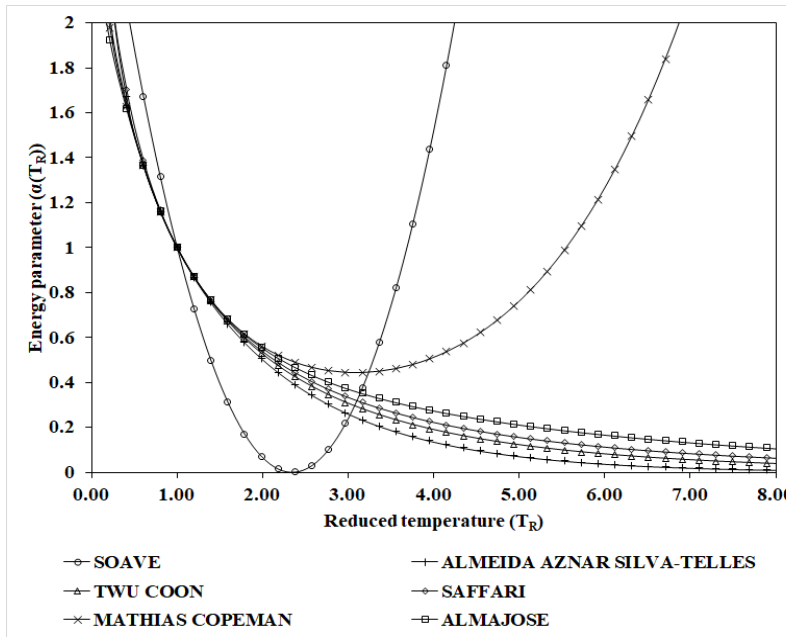

Fig. 1. Behavior of the different alpha functions after fitting water DIPPR vapor pressures.

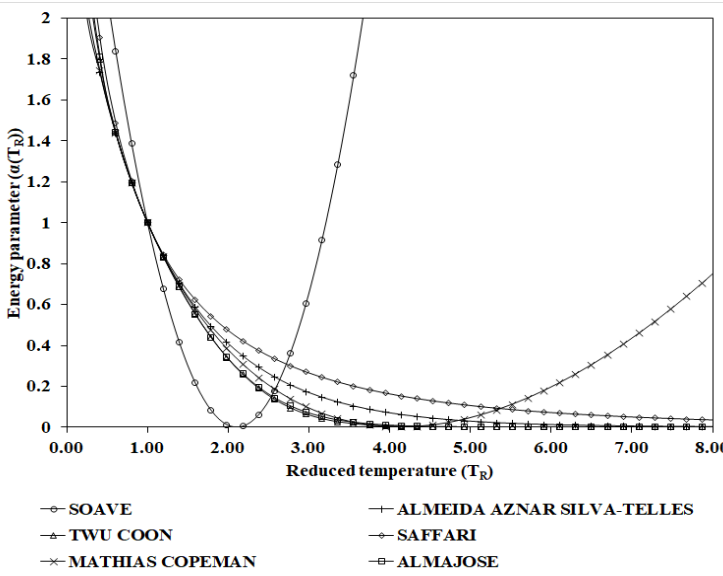

Fig. 2. Behavior of the different alpha functions after fitting octane DIPPR vapor pressures.

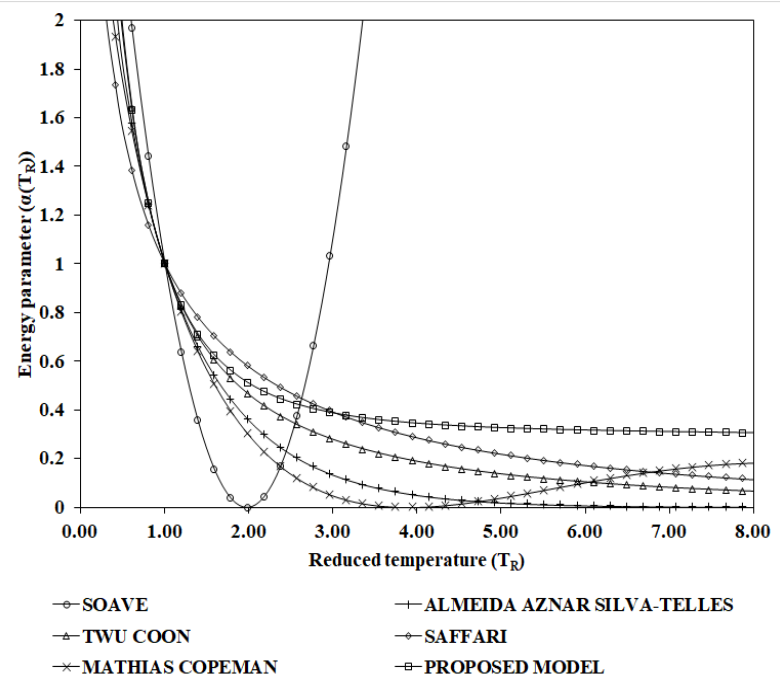

Fig. 3. Behavior of the different alpha functions after fitting ethanol DIPPR vapor pressures.

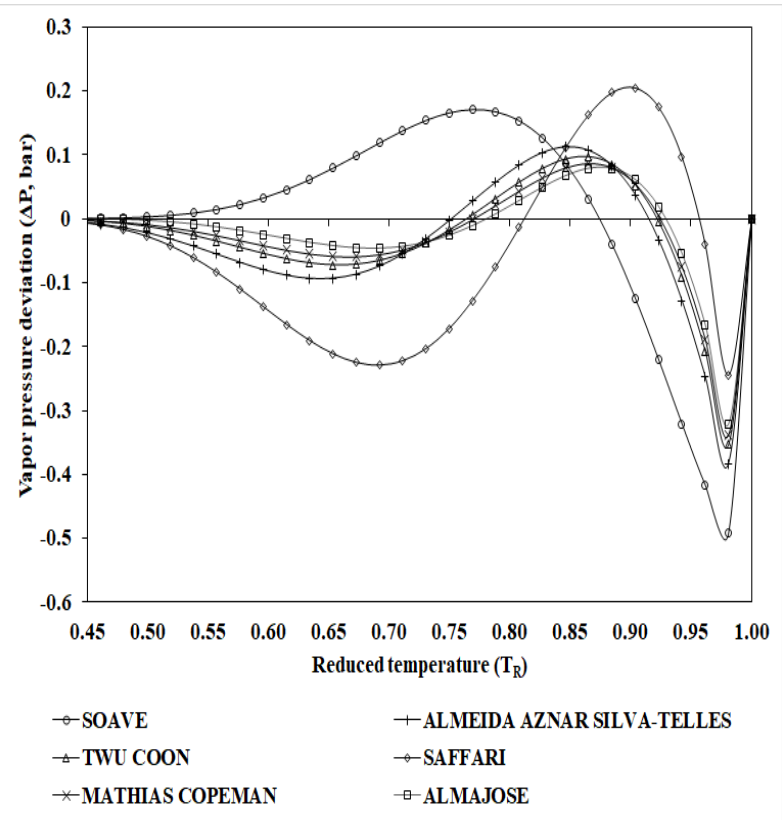

Fig. 4. Vapor pressure deviations for water of each model compared to the DIPPR prediction.

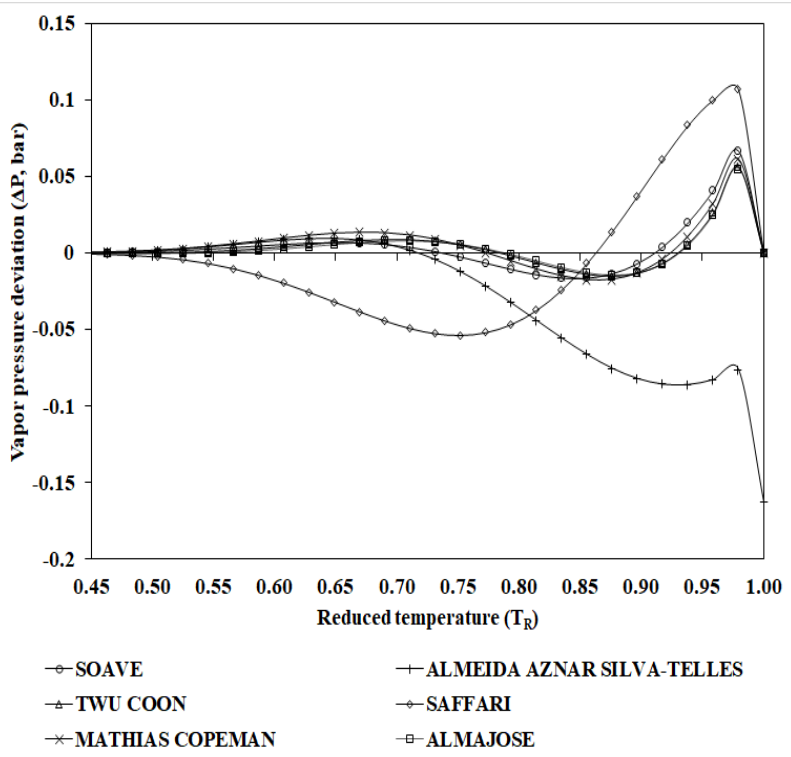

Fig. 5. Vapor pressure deviations for octane of each model compared to the DIPPR prediction. 


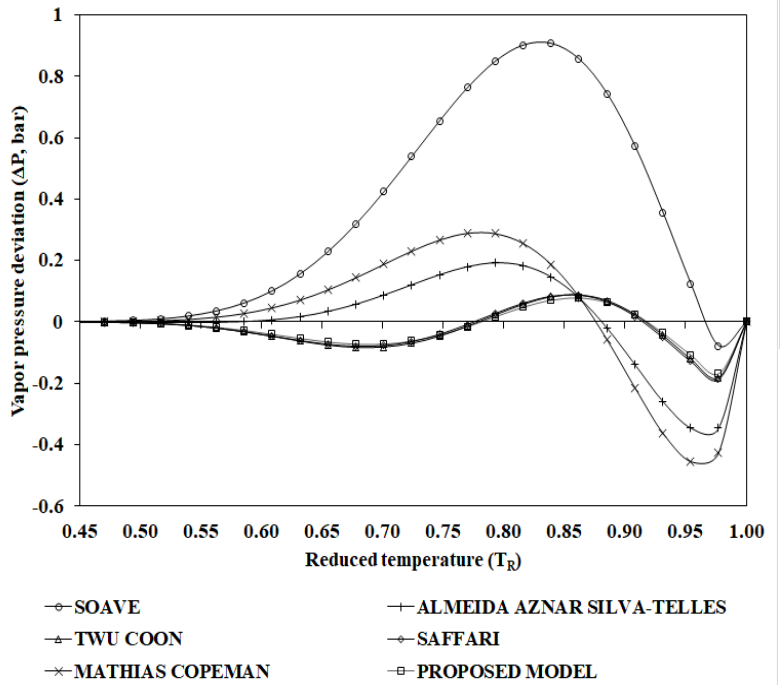

Fig. 6. Vapor pressure deviations for ethanol of each model compared to the DIPPR prediction.
It is of note that the Twu-Coon and the proposed model's performance is very similar, which may be due to the fact that the Twu-Coon is optimized for use on hydrocarbons [6], [7]. The proposed model, while exhibiting minute advantages in accuracy in modelling hydrocarbons and nonpolar compounds, and being comparable in modelling light fluids, exhibit better performance when modelling polar compounds. Fig. 1 to 3 compares the performance of the proposed model to the other models included in this study in modelling vapor pressures for water, octane, and ethanol respectively. Error curves for the three compounds are presented in Fig. 4 to 6.

As an initial listing, thermodynamically consistent parameters for the proposed model have been determined for twenty (20) chemical compounds commonly used in process simulations. Shown in Table II are the constants and the statistical information upon fitting. Constants for other chemical compounds can be made available by request.

TABLE II: PARAMETER LISTING FOR THE PROPOSED ALPHA FUNCTION

\begin{tabular}{|c|c|c|c|c|c|c|c|c|c|}
\hline Compound & A & B & $\mathrm{C}$ & $\mathrm{D}$ & $\mathrm{R}^{2}$ & $\mathrm{R}^{2}$-adj & $\mathrm{R}^{2}$-pred & $\mathrm{AAD}$ & RMS \\
\hline Methane & 0.4061122 & 0.0528853 & 1.3148276 & 0.1217892 & 0.9999948 & 0.9999941 & 0.9999936 & 0.0245580 & 0.0310144 \\
\hline Ethane & 0.4340135 & -0.019447 & 1.2988482 & 0.1265230 & 0.9999951 & 0.9999944 & 0.9999934 & 0.0229178 & 0.0304199 \\
\hline Propane & 0.3191512 & 0.0851615 & 1.5863616 & 0.4135536 & 0.9999904 & 0.9999890 & 0.9999840 & 0.0203792 & 0.0361577 \\
\hline Butane & 0.3577913 & 0.0957190 & 1.5343229 & 0.4528842 & 0.9999931 & 0.9999920 & 0.9999892 & 0.0169816 & 0.0279001 \\
\hline Pentane & 0.3960950 & 0.1528652 & 1.4117964 & 0.5218471 & 0.9999996 & 0.9999995 & 0.9999989 & 0.0031381 & 0.0060610 \\
\hline Heptane & 0.5194211 & 0.2623174 & 1.1731869 & 0.5994893 & 0.9999983 & 0.9999980 & 0.9999964 & 0.0051452 & 0.0098327 \\
\hline Octane & 0.6074828 & 0.3022374 & 1.1088454 & 0.6091066 & 0.9999967 & 0.9999962 & 0.9999947 & 0.0067463 & 0.0125563 \\
\hline Nonane & 0.0000000 & -0.616773 & 1.1342328 & 0.3005279 & 0.9999839 & 0.9999815 & 0.9999785 & 0.0122413 & 0.0254953 \\
\hline Decane & 0.8790198 & 0.5315567 & 0.8717527 & 0.6804762 & 0.9999990 & 0.9999988 & 0.9999981 & 0.0030481 & 0.0059041 \\
\hline Eicosane & 0.1544585 & -0.881831 & 1.0487456 & 0.4215551 & 0.9999446 & 0.9999361 & 0.9999179 & 0.0120278 & 0.0230977 \\
\hline Water & 0.0636178 & -0.406049 & 1.2438683 & 0.2607271 & 0.9999984 & 0.9999981 & 0.9999970 & 0.0490853 & 0.0792088 \\
\hline Acetone & 0.0000000 & -0.336352 & 0.8666082 & 0.3187753 & 0.9999862 & 0.9999840 & 0.9999800 & 0.0289370 & 0.0490548 \\
\hline Ammonia & 0.0000000 & -0.532705 & 1.1315142 & 0.0819312 & 0.9999791 & 0.9999759 & 0.9999744 & 0.0967928 & 0.1508884 \\
\hline Carbon dioxide & 0.6428012 & 0.4488448 & 0.2699924 & 0.5100835 & 0.9999952 & 0.9999945 & 0.9999942 & 0.0263169 & 0.0452421 \\
\hline Ethylene & 0.6465486 & 0.4167281 & 0.4672807 & 0.3364375 & 0.9999948 & 0.9999940 & 0.9999935 & 0.0245921 & 0.0333603 \\
\hline Benzene & 0.4414514 & 0.1687671 & 1.2509208 & 0.4302273 & 0.9999949 & 0.9999941 & 0.9999937 & 0.0263681 & 0.0323067 \\
\hline Styrene & 0.5036085 & 0.1150878 & 1.3215969 & 0.4230738 & 0.9999969 & 0.9999964 & 0.9999962 & 0.0155635 & 0.0189231 \\
\hline Phenol & 1.1293489 & 0.4128892 & 0.8580727 & 0.3440590 & 0.9994891 & 0.9994105 & 0.9993902 & 0.2690559 & 0.3798358 \\
\hline
\end{tabular}

\section{CONCLUSIONS AND RECOMMENDATIONS}

Statistical analyses have proven that the derived alpha function provides better accuracy while being thermodynamically consistent. An alpha function that can be used to predict vapor pressures accurately has been derived, keeping in mind the thermodynamic consistency rules. In order to improve the research, it is recommended to determine the effect in prediction of the proposed alpha function for mixture thermodynamics and supercritical region prediction. While it is assumed that the predictions will improve as suggested by previous researches, quantifiable measurements are desired. Further, it is recommended to utilize a different equation of state and calculate the thermodynamically consistent parameters for the alpha function. While the PTVEOS is used for better accuracy, the Redlich-Kwong and the Peng-Robinson equations are of general utility, and as such, it would be best to determine the alpha function parameters for the two equations of state.

\section{ACKNOWLEDGMENT}

The authors wish to thank the Department of Chemical Engineering of the University of the Philippines - Diliman for giving us the permission to represent the university in the ICCCP 2019. Further, the authors wish to thank the Department of Science and Technology - Engineering Research and Development for Technology for funding this research.

\section{REFERENCES}

[1] S. P. Hosseinifar and S. Jamshidi, "An evolved cubic equation of state with a new attractive term," Fluid Phase Equilibria, pp. 58-71, 2016.

[2] J. O. Valderrama, "The state of cubic equations of state," Industrial Engineering and Chemistry Research, pp. 1603-1618, 2003. 
[3] G. Soave, "Equilibrium constants from a modified Redlich-Kwong equation of state," Chemical Engineering Science, pp. 1197-1203, 1972.

[4] Y. Le Guennec, S. Lasala, R. Privat, and J.-N. Jaubert, "On the imperative need to use a consistent $\alpha$-function for the prediction of pure-compound supercritical properties with a cubic equation of state," Fluid Phase Equilibria, p. 10, 2017.

[5] Design Institute for Physical Properties. (1 June 2018). [Online]. Available: https://www.aiche.org/dippr

[6] C. H. Twu, J. E. Coon, and J. R. Cunningham, "A cubic equation of state with a new alpha function and a new mixing rule," Fluid Phase Equilibria, vol. 69, pp. 33-50, 1991.

[7] C. H. Twu, W. D. Sim, and V. Tassone, "Getting a handle on advanced cubic equations of state," Chemical Engineering Progress, pp. 58-65, 2002.

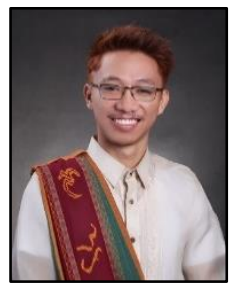

Allan Paolo L. Almajose is a researcher on numerical simulations in chemical engineering. A licensed chemical engineer, educator and programmer, he earned his master's degree in chemical engineering from the University of the Philippines - Diliman last August 2018. He earned units in professional education from the University of the City of Manila last November 2016 and is a chemical engineering graduate of the University of
Santo Tomas (UST) last March 2014. Previously he teaches chemical engineering core course subjects in the Technological Institute of the Philippines, a former teacher for Science - Technology - Engineering Mathematics-related subjects in Arellano University and Jose Rizal University; a former programming consultant in Montessori de San Juan in Manila, Philippines; a former consultant for intermediate to advanced mathematical modeling and numerical simulations in UST Chemical Engineering Department; and a former review specialist for chemical engineering graduates in licensure examinations. Engr. Almajose is currently interested on thermodynamic and mathematical modelling that will optimize PVT relations under the supervision of his adviser and co-author of this paper, Dr. Maria Lourdes P. Dalida.

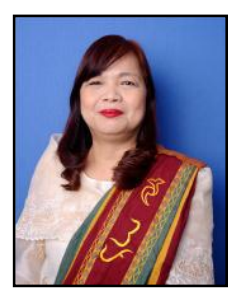

Maria Lourdes P. Dalida is an associate professor at the Department of Chemical Engineering, University of the Philippines Diliman, Quezon City, Philippines. She obtained her Ph.D. in chemical engineering at the University of the Philippines Diliman in 2005 and postdoctoral studies at the University of Houston in 2011. She has over 20 years teaching experience in the said university; teaching chemical engineering core courses and is currently the head of the catalyst research laboratory and bioprocess engineering laboratory of the department. her research interests include photocatalysis, membrane separation processes, boprocessing and wastewater treatment. 